\title{
Mutations Altering the Interplay between GKDnaC Helicase and DNA Reveal an Insight into Helicase Unwinding
}

\author{
Yu-Hua Lo ${ }^{1,2}$, Shih-Wei Liu ${ }^{3}$, Yuh-Ju Sun ${ }^{2}$, Hung-Wen Lizz ${ }^{3 *}$, Chwan-Deng Hsiao ${ }^{1 *}$ \\ 1 Institute of Molecular Biology, Academia Sinica, Taipei, Taiwan, 2 Institute of Bioinformatics and Structural Biology, National Tsing Hua University, Hsinchu, Taiwan, \\ 3 Department of Chemistry, National Taiwan University, Taipei, Taiwan
}

\begin{abstract}
Replicative helicases are essential molecular machines that utilize energy derived from NTP hydrolysis to move along nucleic acids and to unwind double-stranded DNA (dsDNA). Our earlier crystal structure of the hexameric helicase from Geobacillus kaustophilus HTA426 (GkDnaC) in complex with single-stranded DNA (ssDNA) suggested several key residues responsible for DNA binding that likely play a role in DNA translocation during the unwinding process. Here, we demonstrated that the unwinding activities of mutants with substitutions at these key residues in GkDnaC are 2-4-fold higher than that of wildtype protein. We also observed the faster unwinding velocities in these mutants using single-molecule experiments. A partial loss in the interaction of helicase with ssDNA leads to an enhancement in helicase efficiency, while their ATPase activities remain unchanged. In strong contrast, adding accessory proteins (DnaG or Dnal) to GkDnaC helicase alters the ATPase, unwinding efficiency and the unwinding velocity of the helicase. It suggests that the unwinding velocity of helicase could be modulated by two different pathways, the efficiency of ATP hydrolysis or protein-DNA interaction.
\end{abstract}

Citation: Lo Y-H, Liu S-W, Sun Y-J, Li H-W, Hsiao C-D (2011) Mutations Altering the Interplay between GkDnaC Helicase and DNA Reveal an Insight into Helicase Unwinding. PLoS ONE 6(12): e29016. doi:10.1371/journal.pone.0029016

Editor: Arthur J. Lustig, Tulane University Health Sciences Center, United States of America

Received August 22, 2011; Accepted November 17, 2011; Published December 13, 2011

Copyright: (ㄷ 2011 Lo et al. This is an open-access article distributed under the terms of the Creative Commons Attribution License, which permits unrestricted use, distribution, and reproduction in any medium, provided the original author and source are credited.

Funding: This work was supported by research grants from Academia Sinica to C.-D.H. and the National Science Council (NSC98-2311-B-001-009-MY3 to C.-D.H., and NSC98-2113-M-002-013-MY2 to H.-W.L.), Taiwan, Republic of China. The funders had no role in study design, data collection and analysis, decision to publish, or preparation of the manuscript.

Competing Interests: The authors have declared that no competing interests exist.

*E-mail: hsiao@gate.sinica.edu.tw

\section{Introduction}

DNA helicase is a motor protein that unwinds and separates duplex DNA using energy derived from the hydrolysis of nucleoside triphosphates (NTPs). This unwinding activity is ubiquitous in all pathways of DNA metabolism, such as DNA replication, repair and recombination [1,2]. Helicases have been classified into six superfamilies (SF-I to SF-VI) based on conserved sequence motifs characteristic of proteins that catalyze directional translocation on nucleic acids [3,4]. SF-I and SF-II helicases generally operate as monomers or dimers on a diverse range of DNA and RNA substrates, while SF-III to SF-VI helicases adopt hexameric structures and function in replication [4]. In general, the SF-IV hexameric helicases unwind DNA in the $5^{\prime}$ to $3^{\prime}$ direction. The unwinding of duplex DNA by the catalytic action of helicases involves unidirectional translocation and base-pair separation. Since all DNA substrates cannot be unwound within a single biochemical catalytic cycle, the helicase translocation along DNA must involve a series of cyclical binding and release events, which implies that the affinity of helicase for the DNA transiently changes during translocation [5].

Our earlier crystal structural studies of the $G k \mathrm{DnaC}$-ssDNA complex, identified ssDNA-binding pockets on the interior surface of the hexameric ring [6]. The ssDNA-binding pockets are directed toward the N-terminal domain collar of $G k \mathrm{DnaC}$, thus orienting the $5^{\prime}$-end ssDNA toward the DnaG primase to facilitate the synthesis of short RNA primers. We also have previously identified several critical residues that interact with the ssDNA [6]. The locations of these key residues imply that these ssDNAinteracting sites could correlate with helicase translocation. However, unidirectional translocation of the helicase along ssDNA may play a fundamental role in the unwinding process. To understand the hexameric helicase-catalyzed unwinding process in detail, we studied how the helicase and ATPase activities are correlated with the DNA binding. The approach taken is to acquire a detailed knowledge of the structure and the kinetic/ mechanistic information. Recently, single-molecule (sm) techniques, such as total internal reflection fluorescence microscopy (TIRF), single-pair FRET, magnetic/optical tweezers, have been shown to provide novel mechanistic details in helicase action [7]. These single-molecule techniques directly detect the helicase translocation and unwinding in real-time, as well as the conformational dynamics in protein-DNA and protein-protein interactions during helicase action, offer new information that were averaged-out in typical ensemble biochemical experiments. For example, optical and magnetic tweezers-based single-molecule experiments have been used to study several ring-shape helicases, and have determined the force dependence of helicase unwinding kinetics $[8,9,10]$. These studies indicate that phage T7 gp4 helicase unwinds DNA in an active form [8], whereas the phage T4 gp41 helicase unwinds DNA passively [9]. E. coli DnaB helicase was shown by magnetic tweezers experiments that its unwinding rate, translocation rate, and pausing activity can be modulated by force as well as DNA geometry [10]. In addition, complex dynamics in 
replication fork, such as T4 primosome, was also studied by singlemolecule methods to show the coordination among different protein complex during replication [11].

In this study, we use the replicative helicase of Geobacillus kaustophilus, a gram-positive bacterium, as a model system $(G k \mathrm{DnaC})$. To investigate the nature of unwinding by hexameric replicative GkDnaC helicase, we studied how the activity of $G k \mathrm{DnaC}$ is modulated by specific mutations and by accessory proteins. We first compared the unwinding efficiency of these helicases, using gel shift assays, and measured their ATPase activities using spectrophotometric assay. We also developed a single-molecule tethered particle motion (smTPM) experiment to directly determine the unwinding velocity of replicative helicase $G k$ DnaC in real-time. The observation by smTPM had several advantages for this study. First, the method is suited for length and elasticity study of the DNA molecules, which are correlated to the helicase-mediated unwinding processes. Second, the technique allows the study of helicases interaction on torsion relaxed DNA molecules in a nearly force-free experiment. Surprisingly, weakened interaction between helicase and ssDNA leads to an enhancement in helicase efficiency and faster unwinding velocity, while ATPase activities remain unchanged. In strong contrast, adding accessory proteins (DnaG or DnaI) to GkDnaC helicase, alters the ATPase, as well as the unwinding efficiency and the unwinding velocity of the helicase.

\section{Materials and Methods}

\section{Cloning, expression and purification}

Protein expression and purification of $G k$ DnaC wild-type (WT) and mutants were done as previously described [6]. For control experiments, the new constructs GkDnaC K309A and R420A mutants were generated according to the QuikChange mutagenesis protocol (Stratagene, La Jolla, CA) using the pET21b-GkDnaC wild-type plasmid as the template. These mutants were overexpressed and purified similar to WT, and showed identical chromatographic behavior as that of the GkDnaC WT on a size-exclusion column (data not shown). Therefore, amino acids substituted on these mutants do not affect hexameric formation. The coding region of full-length GkDnaG was generated by PCR amplification of genomic DNA isolated from Geobacillus Kaustophilus HTA426 using Pfu DNA polymerase (Stratagene). The forward and reverse primers were designed to incorporate unique NdeI and XhoI restriction sites, respectively, permitting the insertion of the amplified product into the pET21b vector (Novagen) for protein expression in $E$. coli. The resulting plasmid, pET21b-GkDnaG, encodes fulllength wild-type $G k \mathrm{DnaG}$ fused with a $\mathrm{C}$-terminal $\mathrm{His}_{6}$ tag (LEHHHHHH). Escherichia coli BL21(DE3) cells (Yeastern Biotech. Co., Ltd.) were transformed with these expression vectors and grown at $37^{\circ} \mathrm{C}$ in Luria-Bertani medium containing $50 \mu \mathrm{g} / \mathrm{ml}$ ampicillin until the $\mathrm{OD}_{600}$ reached a value of 0.7 . Overexpression of $G k \mathrm{DnaG}$ was induced with $1 \mathrm{mM}$ IPTG for $6 \mathrm{~h}$ at $20^{\circ} \mathrm{C}$. Harvested cells were resuspended in buffer A $(10 \mathrm{mM}$ sodium phosphate, $\mathrm{pH} 7.0)$ and then lysed by sonication. Due to the low binding affinity by His-trap column, GkDnaG proteins were purified from the soluble supernatant on a HiTrap Heparin HP column $(5 \times 5 \mathrm{ml}$, GE Healthcare $)$ followed by purification on a Q-sepharose column (GE Healthcare). The purified proteins were collected and dialyzed against buffer B (10 mM Tris-HCl, pH 8.0, $100 \mathrm{mM} \mathrm{NaCl}$ ). Column fractions were analyzed by SDS-PAGE. Details for construction and protein purification of co-expressed GkDnaCGkDnaI complex have been described previously [12]

\section{ATPase assay}

The ATPase assay is based on a reaction in which ATP hydrolysis is coupled to the NADH oxidation. The kinetics of NADH disappearance was monitored at $340 \mathrm{~nm}$ using a spectrophotometer (Shimadzu UV1800). The assay was performed at room temperature with a reaction buffer containing $50 \mathrm{mM}$ Tris-HCl (pH 7.4), $0.8 \mathrm{mM}$ DTT, $20 \mathrm{mM} \beta$-mercaptoethanol, $0.5 \mathrm{mg} / \mathrm{ml} \mathrm{BSA}$ and $5 \mathrm{mM} \mathrm{MgCl}_{2}$. The reaction mixture was supplemented with $11.36 \mathrm{U} / \mathrm{ml}$ phosphoenolpyruvate (PEP), $20 \mathrm{U} / \mathrm{ml}$ pyruvate kinase (PK), $20 \mathrm{U} / \mathrm{ml}$ L-lactate dehydrogenase $(\mathrm{LDH}), 0.08 \mathrm{mg} / \mathrm{ml} \mathrm{NADH}, 5 \mathrm{mM}$ ATP, and $1 \mu \mathrm{M}$ GkDnaC helicase (or GkDnaC mutants) in the absence or presence of 15mer single-stranded oligo(dT) $(50 \mathrm{nM})$ in a final volume of $150 \mu \mathrm{l}$. All chemicals were purchased from Sigma. Experiments using longer DNA (30- and 70-nt) substrates yielded similar ATPase rates (Figure $\mathrm{S} 1$ ). The rate of ATP hydrolysis is proportional to the rate of the decrease in absorbance at $340 \mathrm{~nm}$ and is calculated according to the formula: $\Delta \mathrm{A}_{340} /$ time $\left(\mathrm{s}^{-1}\right) \times 9820=$ rate of ATPase $(\mu \mathrm{M} / \mathrm{min})$ [13].

\section{Gel shift assay}

A 60-nt-long oligonucleotide (5'-ACATGATAAG ATACATGGAT GAGTTTGGAC AAACGACAAC GTAAAAGGAC GGCCAGTGCC-3', Mission Biotech) at $1 \mathrm{mM}$ was biotinlabeled at the $3^{\prime}$ end using terminal deoxynucleotidyl transferase (TdT, Thermo) and then annealed to an equal molar ratio of M13mp18 single-stranded circular DNA (NEW ENGLAND, BioLabs). M13mpl8 vector is derivative of the single-stranded, male-specific filamentous DNA bacteriophage M13 and it is $7249 \mathrm{bp}$ in length. The mixture was heated at $95^{\circ} \mathrm{C}$ for $2 \mathrm{~min}$, then allowed to anneal at $65^{\circ} \mathrm{C}$ for $20 \mathrm{~min}$, and cooled down slowly to room temperature. The $3^{\prime}$-terminal region (20 nt) of the oligonucleotide is complementary to M13mp18 ssDNA, while the remaining region forms a long overhang tail to create a replication fork-like template. The DNA substrates were purified on a Sepharose CL-4B spin column according to the protocol for separation and isolation of small and large DNA fragments [14]. DNA-unwinding activity assays with $G k$ DnaC helicase were carried out using an ECL-EMSA kit (enhanced chemiluminescence electrophoretic mobility shift assay, Thermo). Approximately $125 \mathrm{nM}$ of the fork-DNA substrate was incubated at $37^{\circ} \mathrm{C}$ for $40 \mathrm{~min}$ with the $\mathrm{GkDnaC}$ proteins $(1 \mu \mathrm{M}$ refer to monomer $)$ in $50 \mathrm{mM}$ Tris-HCl (pH 7.4) buffer containing $20 \mathrm{mM} \beta$-mercaptoethanol, $5 \mathrm{mM} \mathrm{MgCl}_{2}, 5 \mathrm{mM} \mathrm{ATP}$ and $0.5 \mathrm{mg} / \mathrm{ml}$ bovine serum albumin. The reaction was terminated by adding $5 \mu \mathrm{l}$ of $5 \mathrm{X}$ stop solution containing $0.04 \%$ SDS, $8 \%$ glycerol and $40 \mathrm{mM}$ EDTA (pH 8.0). The amount of junction dissociation was analyzed by electrophoresis using a $10 \%$ native polyacrylamide gel run in $0.5 \mathrm{x}$ TBE for $2 \mathrm{~h}$ at $100 \mathrm{~V}$. The biotin-labeled DNA was then transferred to a positive nylon membrane, UV crosslinked, probed with streptavidin-HRP (horseradish peroxidase) conjugate and incubated with the chemiluminescent substrate. The membrane was then exposed to X-ray film for quantification in an AlphaImager 2200 gel documentation system. For all reactions, unwinding efficiency was defined as the fraction of the unwound ssDNA fragment over all biotin-labeled signals. To normalize these values, the percentage of product was calculated using the equation, $\%$ Unwound $=\left(\% \mathrm{U}_{\mathrm{S}}-\% \mathrm{U}_{0}\right) /\left(\% \mathrm{U}_{100}{ }^{\circ} \mathrm{C}-\% \mathrm{U}_{0}\right)$ where $\%$ Us represents the percentage unwound in the sample lane of interest, $\% \mathrm{U}_{0}$ is the percentage unwound in the unreacted substrate and $\% \mathrm{U}_{100^{\circ} \mathrm{C}}$ is the percentage unwound in substrate treated at $100^{\circ} \mathrm{C}$. Each experiment was performed at least in triplicate. This gel shift assay compares the helicase unwinding 
efficiency by quantifying the amount of DNA unwound at a given time interval for wild-type and mutant helicases.

\section{Single-molecule TPM measurements: DNA substrates}

In order to observe the enzyme unwinding process at the singlemolecule level, we designed a fork DNA substrate called "forkAC90". The DNA substrate consists of three annealed oligonucleotides. Oligonucleotide A is a $145 \mathrm{nt}$ TG-rich strand (5'TTTTTTTTTT TTTTTGAAGT CACAGAAAAG CATCTTATGT GACGGTGTCT GTGTGCTGGT GGGTGTGTGT GTGCTGGTGG GTGTGTGTGT GCTGGTGGGT GTGTGTGTGT GTGTGTGGAG GTGTAGACTA CAGGGTGAGC TATGA, Sigma). This TG-rich strand A was then annealed to two other oligonucleotides to create a nicked substrate: one is an 18 nt-long, 5' -end digoxigenin-labeled oligonucleotide B (5'-digTCATAGCTCACGCTGTAG, Sigma), and the other is a 90 ntlong, 3'-end biotinylated oligonucleotide $\mathrm{C}$ carrying $\mathrm{AC}$ repeats (AC90). The annealed DNA substrate contains a 37 nt singlestranded overhang at its 5 '-end for helicase loading and can be bound to streptavidin-coated beads (200 nm, Bangs Lab, Fishers, IN) through the biotin-labeled end for single-molecule tracking. The AC repeat sequence was designed to avoid potential secondary structure formed during the helicase unwinding. The annealed DNA substrates were purified from excess individual free oligonucleotides using a PCR purification kit (Qiagen). To mimic the unwound product, we also prepared another DNA substrate similar to fork-AC90 substrates except $108 \mathrm{nt}$ from the 5 ' end of oligonucleotide A was removed, leaving a $37 \mathrm{nt}$ long oligonucleotide.

\section{Imaging acquisition and analysis}

The Brownian motion (BM) of tethered beads was observed by means of an inverted optical microscope (Olympus IX-71, Tokyo, Japan), using a Newvicon camera (NC-70, DAGE-MTI, Michigan, IN) by the differential interference contrast (DIC) method at $30 \mathrm{~Hz}$ [15]. The images were directly digitalized through a framegrabber (PCI-1411, National Instrument, Austin, Texas) and stored in a computer. For time-course studies, the amplitude of bead BM was quantified using the mean-squared displacement (MSD; $\left.\left.<\Delta \mathrm{x}^{2}\right\rangle=\left\langle\mathrm{x}^{2}\right\rangle-<\mathrm{x}\right\rangle^{2}$ ) of drift-correlated centroid positions of the tether beads from 20 consecutive frames $(0.66 \mathrm{~s})$. For population distribution measurements, MSD values were calculated from 1000 consecutive frames to obtain the average BM amplitude. To ensure that only one DNA molecule was attached to each bead, only the beads with symmetrical BM were included in analysis, and the ratio of MSD values of $\mathrm{x}$ position and those of y position of the beads was limited to $1.1-0.9[16,17,18]$.

\section{DnaC helicase unwinding experiments by smTPM}

The preparation of the slide chamber and streptavidin-coated beads have been described previously [15]. The fork-AC90 DNA substrate was anchored on an anti-digoxigenin coverslip through its digoxigenin label. After $30 \mathrm{~min}$ incubation at room temperature, the unbound DNA was removed. Then streptavidin-coated polystyrene beads were flowed into the reaction chamber to attach onto the biotin group in the fork-AC90 substrate. After removal of excessive beads, $G k$ DnaC helicase was introduced to load onto the fork-AC90 substrates through its distal $5^{\prime}$-overhang in the presence of ATP to initiate unwinding. The $30 \mu \mathrm{l}, 500 \mathrm{nM}$ enzyme ( $G k$ DnaC helicase, GkDnaC-GkDnaG complex, GkDnaC$G k$ DnaI complex and $G k$ DnaC mutants) in the reaction buffer (50 mM Tris-HCl, pH 7.4, 5 mM MgCl 2,2 mM $\beta$-mercaptoethanol, $5 \mathrm{mM}$ ATP with PK-PEP regeneration system, $2 \mathrm{mg} / \mathrm{ml}$ BSA) was flowed into the slide chamber at $22 \pm 1^{\circ} \mathrm{C}$. Further reduction of enzyme concentration greatly reduced the observed unwinding probability. Reactions using higher enzyme concentration $(10 \mu \mathrm{M})$ returned the same unwinding velocity (data not shown). Thus, it is most likely that a single hexameric DnaC unwinds the DNA substrate at this concentration. The DNA substrate in the unwinding experiment was designed so that the dissociation of the bead-labeled oligonucleotide AC90 signals the completion of unwinding of the $90 \mathrm{bp}$ duplex region. During the unwinding of the $90 \mathrm{bp}$ duplex substrates, the conversion of duplex into single strands lead to an increase in BM, as confirmed by the BM increase from the fork-AC90 substrate to the mimicked unwound substrate. The Gaussian fitting of the forked $(\mathrm{N}=66)$ and mimicked unwound $(\mathrm{N}=57)$ substrates returned with a mean \pm standard deviation of the distribution. The change in the $\mathrm{BM}$ of the beads thus correlates with the GkDnaC-mediated unwinding kinetics. The conversion factor is based on the difference BM between these two substrates and their difference on duplex length, and is determined to be $0.0718 \pm 0.0257 \mathrm{~nm} / \mathrm{bp}$. Only the beads with increasing BM pattern prior to dissociation are included in unwinding velocity analysis. Since the tethers appeared in the absence of $G k \mathrm{DnaC}$, we identified the time point where $G k \mathrm{DnaC}$ binds and the unwinding starts by the changing point in the first derivative of the BM time-course (as shown in Figure S2). Once the initial unwinding time point is identified, the unwinding velocities were determined by applying least-squares linear fits to the increasing BM section of the BM time-courses. The unwinding velocities were also determined using the dwell time between the beginning of $\mathrm{BM}$ increase and the maximum $\mathrm{BM}$ achieved, required to unwind the 90 bp duplex DNA. Both methods yielded the same unwinding velocities within experimental errors.

\section{Bootstrap estimation for Unwinding velocities}

The histograms of mean unwinding velocities were supplied by bootstrapping estimation that resamples the means of the unwinding velocities 100 times. The bootstrapping estimation was executed by R-software (http://www.r-project.org/). We used the ordinary nonparametric bootstrapping method to generate the t-distributions of unwinding velocities based on 10-18 smTPM measurements.

\section{Results and Discussion}

\section{Helicase activity alters as helicase loses partial DNA-binding interaction}

Based on the crystal structure of the GkDnaC-ssDNA complex and biochemical studies on the mutant proteins, we have previously identified several key residues of $G k \mathrm{DnaC}$ involved in ssDNA binding [6]. Previous studies showed that mutations of residues located within the inside channel of the helicase and involved in ssDNA-binding (i.e., K50A, R117A, R120A, R145A, R145A/K146A, R330A, R332A and R344A) increased the dissociation constants of the resulting enzymes by two to three orders of magnitude as compared to that of the wild-type $G k \mathrm{DnaC}$ helicase [6]. We also presented the sequence alignment for these residues in the ssDNA-binding region of $G k \mathrm{DnaC}$ helicase among DnaB-like family, as shown in Figure S3. According to the alignment analysis, some residues are completely conserved among family members (such as R117, R344 and R420), while others belong to a nearly identical or similar group (R45, R120, R145, K146, K309, R330 and R332). We thus suggest that these residues that located at the central channel of helicase play key roles for DNA-binding among DnaB-like family members, which in turn can affect DNA translocation during the unwinding process. To 
test if these mutations indeed alter helicase unwinding, we analyzed the $G k$ DnaC helicase unwinding efficiency by monitoring the amounts of the helicase-catalyzed displacement of short biotinlabeled oligonucleotides from the forked DNA substrates. We first compared the amount of unwinding products generated by wildtype and mutant helicases within a given reaction time using gel shift assay. To optimize experimental sensitivity for the gel shift assay, we first identified the unwinding product as a function of enzyme concentration in a fixed reaction time of 1 hour at the constant amount of DNA substrates. Using increasing amounts of wild-type $G k \mathrm{DnaC}$ WT $(0.1$ to $4 \mu \mathrm{M})$, we found that the relative unwinding efficiency was essentially proportional to the amount of enzyme and displayed a linear gradient up to $1 \mu \mathrm{M}$, and reached the plateau when enzyme concentration is higher than $1 \mu \mathrm{M}$ (Figure S4). We then carried out the gel shift assay for monitor the unwinding efficiency of wild-type and mutant helicases at $1 \mu \mathrm{M}$ protein concentration at $37^{\circ} \mathrm{C}$ for slightly shorter time of 40 minutes (Figure 1A). Interestingly, most $G k$ DnaC mutants in which a positively charged side chain within the center channel had been replaced by an alanine residue showed a significantly enhanced helicase activity (Figure 1A). Among these mutants, the R332A mutant showed a nearly 4-fold increase in helicase unwinding efficiency, suggesting that the DNA-binding loop I (residues 321 to 335, shown in Figure 1B) located in the inside channel of the hexameric helicase is not only responsible for DNA binding but also affects significantly helicase unwinding and/or translocation. By contrast, mutation of a positively charged residue (K309A) located on the outside surface of the hexameric ring and away from the DNA-binding channel (Figure 1B) did not affect the unwinding efficiency of the enzyme (Figure 1C). As a control, mutation of the arginine $(\mathrm{R} 420 \mathrm{~A})$ residue located in the nucleotide-binding pocket from the adjacent subunit rendered the enzyme completely inactive (Figure $1 \mathrm{~B}$ and $1 \mathrm{C}$ ). This control experiment indicated that the mutation in the ATP-binding site of $G k \mathrm{DnaC}$ abolishes the helicase function. These results suggest that partial loss of the interaction between the helicase and ssDNA can alter the helicase unwinding activity. From the structural point of view, these key residues all located in central channel of helicase, we thus considered that protein-ssDNA interaction may correlate with the helicase translocation. Helicase that lost the partial interaction with DNA might translocate faster than before resulting in efficient unwinding. In this weak binding state, helicase could incompletely dissociate from the nucleic acid and easily move forward to the junction.

\section{ATPase activities of wild-type GkDnaC and mutants}

The unwinding activity of helicase requires its coupling with ATP hydrolysis. To understand if the enhanced helicase activity result from differences in ATPase rates of different helicase mutants, we measured the ATPase rates of wild-type $G k \mathrm{DnaC}$ and its mutants. Measurements of ATP hydrolysis were performed both in the absence and presence of ssDNA. The DNAindependent ATPase activity provides information about the intrinsic ability of proteins to hydrolyze ATP, whereas the measurement of ATPase activity in the presence of ssDNA reflects the DNA-stimulated ATPase activity. We carried out a coupled ATPase spectrophotometric assay by monitoring the decrease of $\mathrm{NADH}$ absorbance at $340 \mathrm{~nm}$ (Figure 1D). As expected, the nucleotide-binding pocket mutant R420A showed a low ATPase rate of about $5.7 \mu \mathrm{M} / \mathrm{min}$ (red line, Figure 1D) in the absence of ssDNA, similar to background level of $3.5 \mu \mathrm{M} / \mathrm{min}$ (data not shown). However, for wild-type enzyme and helicase mutants with enhanced activities, the intrinsic ATPase rates are all about $30 \mu \mathrm{M} / \mathrm{min}$. The similar intrinsic ATPase rates of wild-type and mutants indicated that the enhancement of helicase unwinding activity in mutant proteins is not due to an increased efficiency of ATP binding or ATP hydrolysis but resulted from the helicase/ ssDNA interaction. On the other hand, the DNA-stimulated ATPase activities we observed here were only slightly increased as compared to the intrinsic ATPase activity. Although we have used different lengths or different concentrations of ssDNA, the measured ATPase rates are rather similar with the intrinsic ATPase rates (Figure S1 and S5). It is possible that DNA binding does not stimulate ATPase activity in G. kaustophilus hexameric DnaC helicase. Similar observations have been reported for several other hexameric helicases such as BstDnaB, TWINKLE, and $E c o$ RuvB helicases [19,20,21].

\section{Helicase unwinding velocity monitored by smTPM}

To test whether the differences in unwinding efficiencies between the $G k \mathrm{DnaC}$ helicase and its mutants seen in gel shift assays result from the differences in unwinding velocities, we directly measured the unwinding velocities of individual $G k \mathrm{DnaC}$ helicases in real-time at the single-molecule level. Most singlemolecule studies on ring-shaped SF-IV helicases used forcedependent techniques such as optical and magnetic tweezers (in the force range of 5-40 pN) [7,8,9,10,22,23]. Here, we developed a single-molecule tethered particle motion (smTPM) experiment to study the unwinding velocity of $G k \mathrm{DnaC}$ helicase in a nearly forcefree condition (in the force range of $\mathrm{fN}$ ). We tethered the DNA substrates on anti-digoxigenin-decorated coverglass and labeled the distal $5^{\prime}$ end of DNA with streptavidin-coated beads (see Materials and Methods, Figure 2A). The centroid position of the tethered bead can be measured by optical microscopy and determined to nanometer precision by image processing [16]. As unwinding persists, duplex DNA is converted to ssDNA that possesses a higher flexibility. Therefore, a gradual increase in Brownian motion (BM) of the bead is expected from the unwound ssDNA (Figure 2B). The BM of intact, initial fork-AC90 substrates is shown to be fitted into a single Gaussian curve, $9.90 \pm 2.18 \mathrm{~nm}$ (mean \pm standard deviation; $\mathrm{N}=66$, where $\mathrm{N}$ is the number of effective tethers; Figure 2C). To mimic the unwound fork substrate before detachment, we replaced the $145 \mathrm{nt}$ ssDNA with a $37 \mathrm{nt}$ ssDNA annealed to the $3^{\prime}$-end of the biotinylated AC90 ssDNA. The BM of this sample was determined as $15.00 \pm 4.23 \mathrm{~nm}$ (mean \pm standard deviation, $\mathrm{N}=57$ ) as illustrated in Figure 2D. The increased BM reflects the difference between duplex DNA and ssDNA, and confirms that this BM increase can be used to monitor the DnaC-mediated unwinding process with the conversion factor of $0.0718 \pm 0.0257 \mathrm{~nm} / \mathrm{bp}$. The broader BM distribution of this unwound state is consistent with the long singlestranded DNA segment of this substrate.

Upon the addition of $G k \mathrm{DnaC}$ helicase and the mixture of ATP and ATP regeneration system to the coverglass, we observed some tethers with an apparent BM increase followed by tether disappearance (Figure 3A). On average, out of $\sim 30$ initial tethers in the field of view, we observed about 5 tethers $(17 \%)$ that showed this kind of BM increase and tether disappearance. The increase in $\mathrm{BM}$ and tether disappearance suggests that $G k \mathrm{DnaC}$ hexamers alone are capable of unwinding this 90 bp substrate. Using DNA substrates with a longer duplex region, however, did not produce a significant amount of tether disappearance, likely due to the limited processivity of the $G k \mathrm{DnaC}$ helicase. Control experiments without ATP or without $G k \mathrm{DnaC}$ did not render any tether disappearance in BM time-courses (data not shown). There is a recording dead time of about $20 \mathrm{~s}$ due to solution exchange and stage restabilization for imaging (illustrated by the gray-shaded region in Figure 3A, inset). This $\sim 20 \mathrm{~s}$ dead time does not 
A
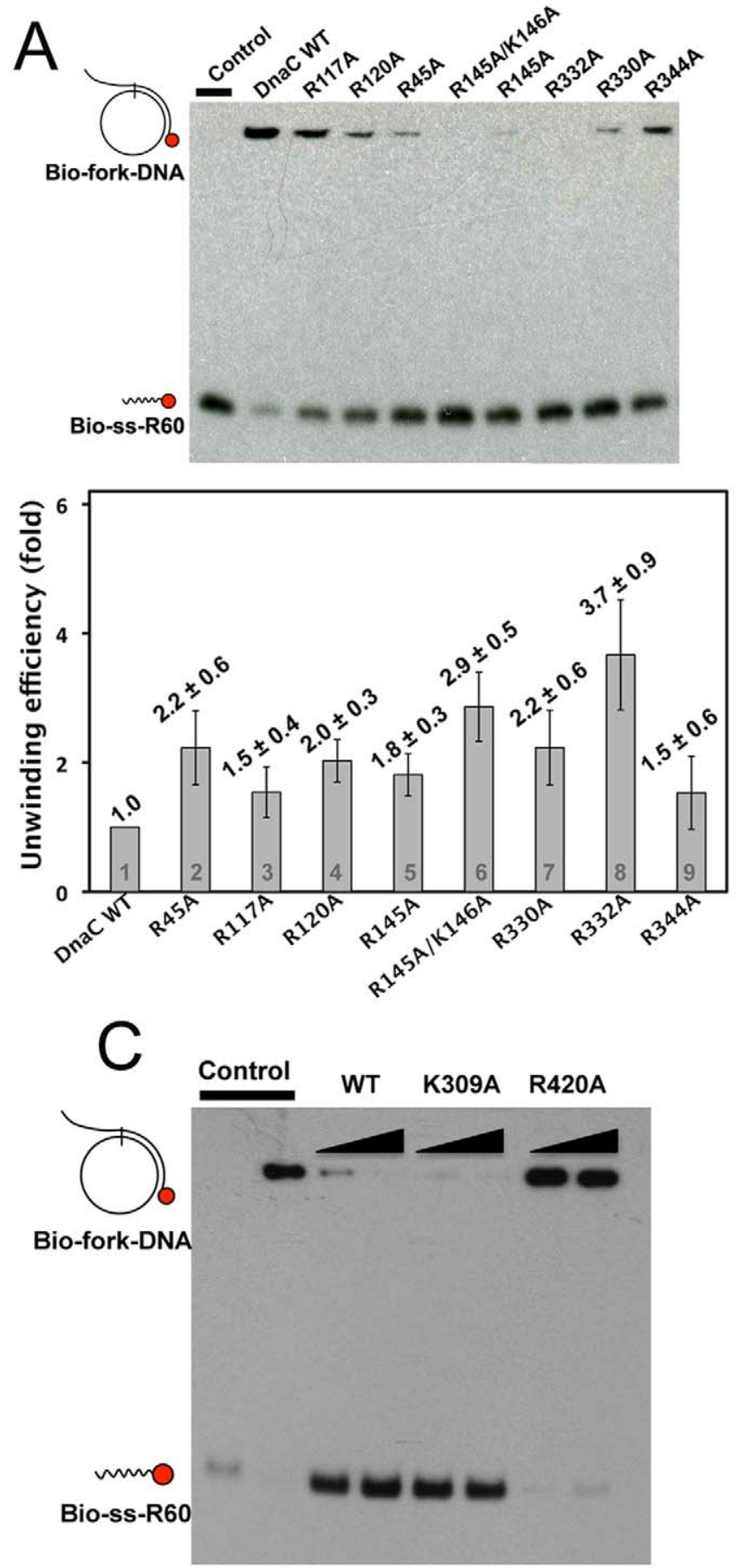
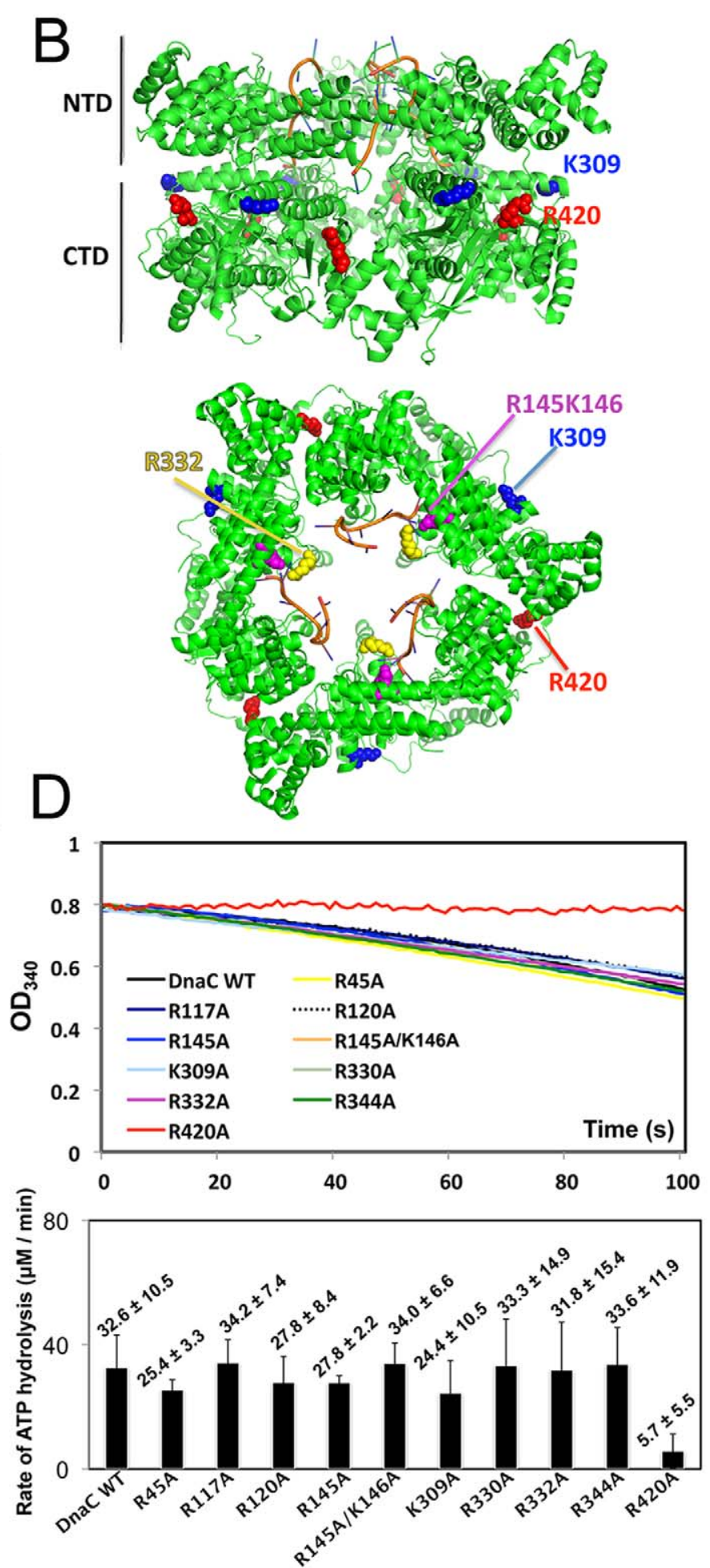

Figure 1. Ensemble assays of wild-type and mutant GKDnaC. A. Gel shift assay of GkDnaC WT and mutants. The enhancement of unwinding efficiency is expressed by the fold of change in the biotin-labeled ssDNA unwound product from GkDnaC mutant to that from WT. Fork-DNA substrate and ssDNA product are indicated schematically on the left. The relative amount of unwinding efficiency of each mutant with reference to GkDnaC WT is indicated. The red sphere denotes the biotin-labeled end. The average and standard deviations of three independent experiments are given. B. Locations of amino acid residues in GkDnaC that were mutated. The hexameric GkDnaC and ssDNA are represented as cartoons and colored in green and orange, respectively. C. Gel shift assay of GkDnaC WT, mutant K309A and R420A (arginine finger). An increasing amount of purified protein $(0.5$ to $1 \mu \mathrm{M})$ was used in the standard gel shift assay. D. Time-courses of ATPase activity of GkDnaC WT and mutants. Reactions were initiated by the addition of $1 \mu \mathrm{M}$ enzyme (per monomer) after preincubation of all other components at RT for 5 min. ATPase rates of WT and mutants are compared. Data represent the average and standard deviations from three independent experiments.

doi:10.1371/journal.pone.0029016.g001 

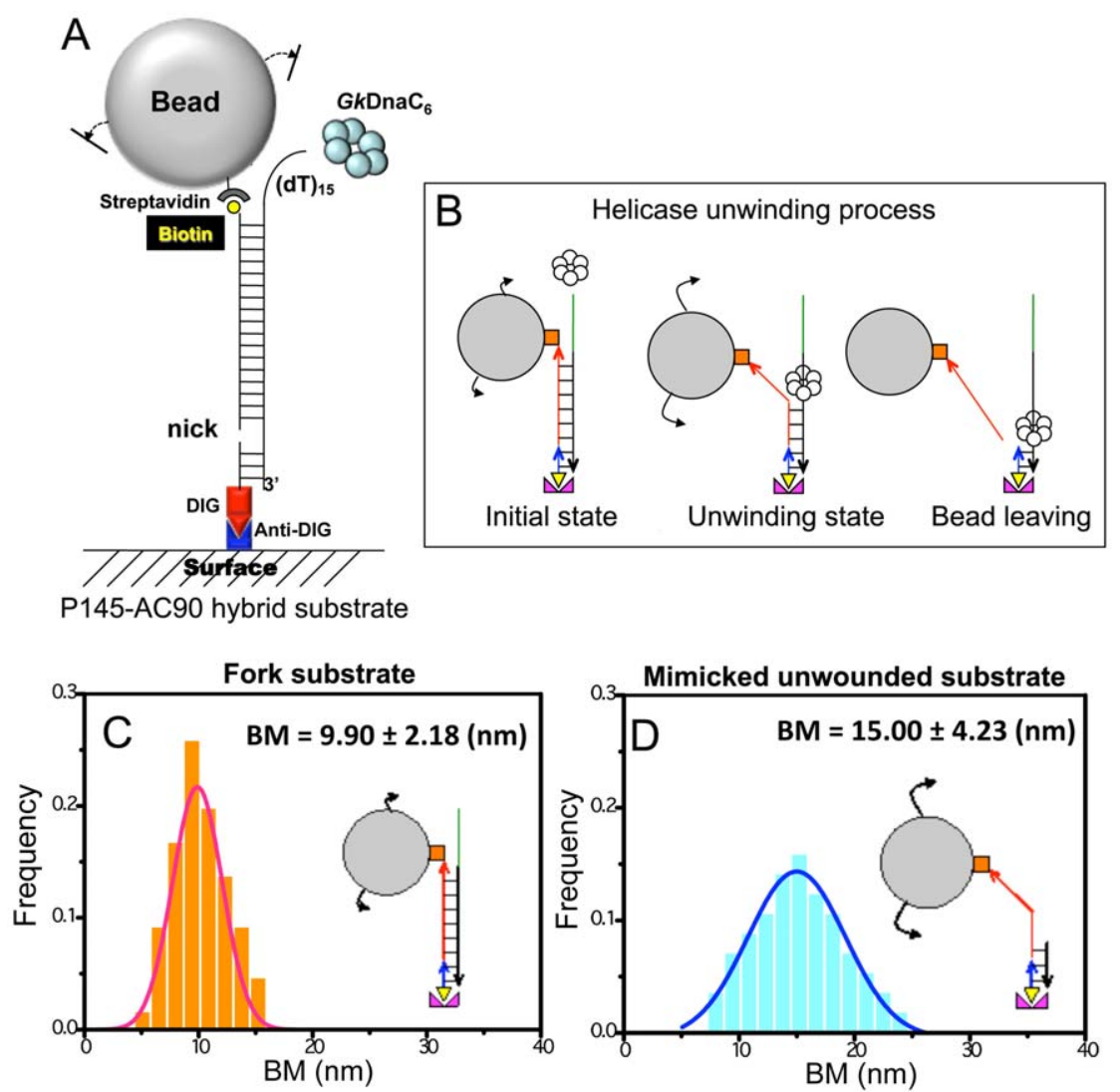

Figure 2. Single-molecule TPM to monitor GKDnaC unwinding. A. Experimental geometry for monitor unwinding velocity. B. GkDnaCmediated unwinding leads to the conversion of duplex DNA into ssDNA, which is signaled by an increase in the BM of the bead, and eventually followed by bead-ssDNA disappearance. In the absence of helicase, a smaller, constant BM amplitude corresponding to the duplex substrate was observed. As GkDnaC binds and initiates unwinding, BM starts to increases. Due to the design of the nicked substrate, the ssDNA-tethered bead disappears and signals the completion of unwinding of the $90 \mathrm{bp}$ duplex. C. The initial BM distribution of the fork substrate with 108 bp duplex DNA and $37 \mathrm{nt} s s D N A$ overhang allowing for $G k D n a C$ binding is $9.90 \pm 2.18 \mathrm{~nm}$ (mean \pm s.d., $\mathrm{N}=66$ ). D. The BM for the mimicked unwound product before bead disappearance. The substrate contains a $37 \mathrm{bp}$ duplex region with a nick and a $71 \mathrm{nt}$ ssDNA. The BM increases to $15.00 \pm 4.23 \mathrm{~nm}$ (mean \pm s.d., $\mathrm{N}=57$ ). It is feasible to monitor the helicase unwinding using the $\mathrm{BM}$ increase. doi:10.1371/journal.pone.0029016.g002

interfere with our observation of GkDnaC-mediated unwinding process, since the bead BM remains constant for at least $>100$ seconds before the unwinding occurs. Only the beads with increasing $\mathrm{BM}$ pattern prior to dissociation are included in unwinding velocity analysis. For these tethers, the increasing BM time-courses can be fitted nicely by a linear line, where the slope of the fit returns the unwinding velocity of individual helicase molecule. The dwell time between the start of BM increase and the maximum BM is the time required to unwind the $90 \mathrm{bp}$ duplex, and also allows the calculation of unwinding velocities. Both methods (slope in linear fits and dwell time) return with similar unwinding velocities. The average unwinding velocity of wild-type $G k \mathrm{DnaC}$ helicase was $3.58 \pm 1.62 \mathrm{bp} / \mathrm{s}(\mathrm{N}=10)$ in this nearly forcefree experiment. This unwinding velocity is slower than that of other ring-shape helicases determined so far, such as bacteriophage T4 gp41 ( $\sim 30 \mathrm{bp} / \mathrm{s}$, the value estimated from extrapolating to zero force), T7 gp4 (29 bp/s at $5.2 \mathrm{pN}, 220 \mathrm{bp} / \mathrm{s}$ at $11.2 \mathrm{pN})$ and Escherichia coli DnaB (80 $50 \mathrm{bp} / \mathrm{s}$, the value estimated from extrapolating to zero force) $[8,9,10]$. In some cases $(<10 \%)$, there is a pause before tethers disappear, likely due to the potential reannealing of the unwound ssDNA to its complementary strand. These tethers were not included in the unwinding velocity analysis.

To further confirm that the increase in BM time-course indeed reflects helicase-catalyzed unwinding processes, we measured the unwinding velocities at different ATP concentrations. Even though hexamer helicases hydrolyze a number of ribonucleoside and deoxyribonucleoside triphosphates (rNTPs and $\mathrm{dNTPs}$ ) in the absence of DNA substrates, their unwinding efficiency is powered by its ATPase, and is thus ATP dependent. We measured the unwinding velocity of $G k \mathrm{DnaC}$ at 5 different ATP concentrations, and determined its unwinding velocities, as shown in Figure 4 and Table 1. The Michaelis-Menten fit yielded values of $V_{\max }$ of $4.69 \pm 2.00 \mathrm{bp} / \mathrm{s}$ and $K_{\mathrm{M}}$, ATP of $1.03 \pm 0.46 \mathrm{mM}$. The observed ATP dependence provides direct and strong evidence that the detected increase in BM time-course describes the GkDnaCcatalyzed unwinding process.

Helicase-DNA interaction is correlated with its unwinding

Our gel shift results suggested that residues located at the inside channel of the helicase that participate in ssDNA binding could affect the helicase unwinding efficiency. The enhanced helicase efficiency of the enzymes carrying a single point mutation at these sites might lead to a faster unwinding velocity. To confirm the results from the gel shift assay, we performed single-molecule timecourse analyses. The increasing BM time-course was fitted to yield an unwinding velocity for each helicase molecule (Figure 3). Exemplary BM time-courses of wild-type and mutant helicases are shown in Figure 3 together with the compiled histograms 


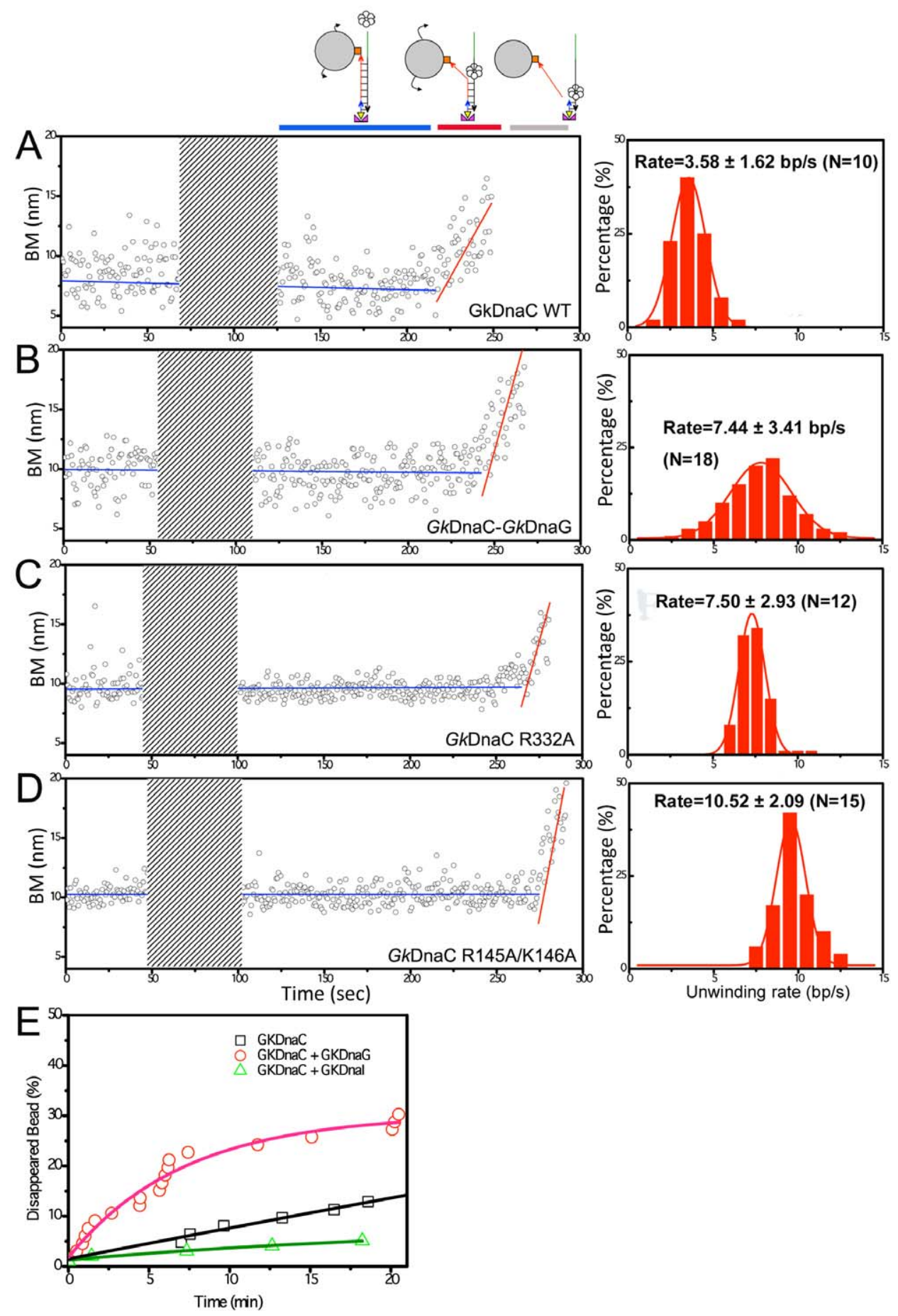

Figure 3. Observed unwinding by an individual $G k D n a C$ enzyme in a smTPM experiment. BM time-course of a single duplex DNA molecule in the presence of the indicated enzyme(s) (left panel) and the histogram of observed unwinding velocities (right panel). A. BM time-course of a single duplex DNA molecule unwound by GkDnaC WT. There is a recording dead time of at least $20 \mathrm{~s}$ due to solution exchange and stage restabilization for imaging (shaded area). The increased slope of BM time-course was fitted to yield an unwinding velocity of $3.59 \pm 1.30 \mathrm{bp} / \mathrm{s}$ for this molecule. Histogram supplemented by data from bootstrapping statistics based on the mean of the measured unwinding velocities, shows the unwinding velocity (mean \pm s.d., $\mathrm{N}=10$ ). B-D. BM time-course for GkDnaC-GkDnaG complex, GkDnaC mutant R332A and R145A/K146A. The unwinding velocities of individual traces are $7.33 \pm 2.63,7.30 \pm 2.66$, and $10.56 \pm 3.82 \mathrm{bp} / \mathrm{s}$, respectively. The mean unwinding velocities are shown in the histogram. E. Time-course of disappeared tethered beads (in percent) observed by single-molecule TPM experiments. The cumulative exponential curve was fitted with the equation: $y=y_{0}+\left[1-(1 / t) e^{(-x / t)}\right]$. doi:10.1371/journal.pone.0029016.g003

supplemented with bootstrapping analysis. As shown in Figure 3C and 3D, the mean unwinding velocities of R145A/K146A and R332A mutants were found to be $10.52 \pm 2.09 \mathrm{bp} / \mathrm{s}$ (R145A/
$\mathrm{K} 146 \mathrm{~A}, \mathrm{~N}=15)$ and $7.50 \pm 2.93 \mathrm{bp} / \mathrm{s}(\mathrm{R} 332 \mathrm{~A}, \mathrm{~N}=12)$, respectively. Both mutants showed apparent, statistically significant, increased unwinding velocities as compared to the wild-type 


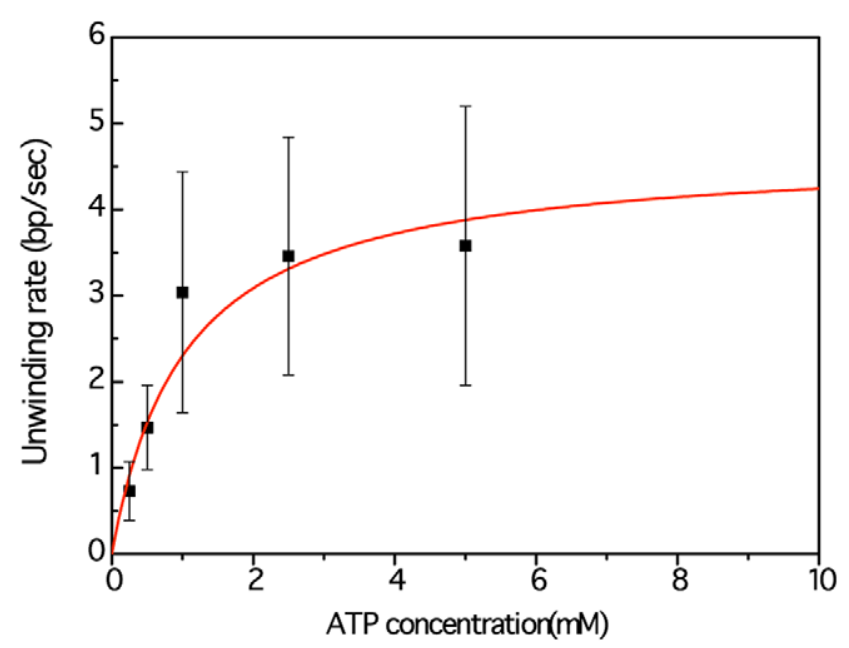

Figure 4. ATP dependence of unwinding velocities of GKDnaC WT obtained from SmTPM. Helicase unwinding velocities were measured at different ATP concentrations $(0.25-5.0 \mathrm{mM})$. The data were fitted to Michaelis-Menten kinetics, yielding $K_{\mathrm{M}}$, ATP of $1.03 \pm 0.46 \mathrm{mM}$ and $V_{\max }$ of $4.69 \pm 2.00 \mathrm{bp} / \mathrm{s}$.

doi:10.1371/journal.pone.0029016.g004

enzyme $(3.58 \pm 1.62 \mathrm{bp} / \mathrm{s})$, with the consideration of the error associated with the conversion factor. In addition, in the BM timecourses of mutants and the wild-type helicases, no apparent backstep motion was observed in the limited resolution of the TPM experiments. As summaried in Table 2, enhanced unwinding velocities (and unwinding efficiency) of these helicase mutants are not due to an increase of their ATPase activity, since their ATPase rates are basically similar to that of the wild-type enzyme. Based on kinetic evidence at the single-molecule level and structural analysis in a previous study [6], we speculated that helicase translocation and unwinding can be effectively modulated by its binding affinity towards ssDNA. This can be achieved by direct alteration of the helicase itself as shown in our mutation studies. Previous studies reported that the unwinding velocities of hexameric helicases have a strong force dependence $[8,9,10]$. Although the applied force mainly modulates the stability of DNA substrate, but the external forces may also influence the helicaseDNA interaction resulting in an efficient unwinding action.

Based on structural analysis [6], we identified that Arg145 interacts with the ribose of nucleotide through hydrogen bonding, whereas Lys146 binds in the same manner to the base of nucleotide. In the CTD (G-terminal domain) collar, Arg332 is located in the DNA-binding Loop I which is structurally conserved in RecA-like family [6,24]. The Loop I (A) and Loop I (B) from both subunits buttress the $3^{\prime}$-end of ssDNA, and these flanking

Table 1. ATP dependence of the unwinding velocity measured by smTPM.

\begin{tabular}{ll}
\hline ATP conc. (mM) & Unwinding velocity (bp/s) \\
\hline 5.0 & $3.58 \pm 1.62(\mathrm{~N}=15)$ \\
2.5 & $3.46 \pm 1.45(\mathrm{~N}=10)$ \\
1.0 & $3.04 \pm 1.40(\mathrm{~N}=10)$ \\
0.5 & $1.47 \pm 0.57(\mathrm{~N}=5)$ \\
0.25 & $0.73 \pm 0.34(\mathrm{~N}=10)$ \\
\hline doi:10.1371/journal.pone.0029016.t001
\end{tabular}

loops function like a clamp to mediate the ssDNA binding via hydrogen bonds and a salt-bridge to the phosphate backbone. Because that hydrogen bonding interaction plays a critical role between protein and DNA, changing these positively charged residues (Arg145, Lys146 and Arg332) to alanine alters the protein-DNA interaction significantly. In addition, previous studies showed that the E. coli DnaB hexamer could occlude 20mer ssDNA in length, but only 10-mer ssDNA was strongly protected against nuclease digestion [25,26,27]. We also only observed 9-mer ssDNA seating on the basic DNA-binding pocket formed from two subunits in the asymmetric unit, although we used 15-mer oligo(dT) for crystallization [6]. Therefore, in $G$. kaustophilus, we suggested that the DNA-binding site we found in NTD (N-terminal domain) collar probably belongs to a "strong", DNA-binding site. However, in the cases of the papillomavirus E1 helicase and $E$. coli Rho helicase (smaller diameter in central channel of 17 20 $\AA$ ), nucleic acid is bound within the channel via loops that form a "spiral staircase" protruding from each subunit $[28,29]$. We can thus speculate that two DNA-binding sites exist in the central channel of hexameric GkDnaC: one located in the wider NTD collar of $50 \AA$ in diameter ("strong" DNA-binding site) and the other in the narrow CTD collar of $\sim 20 \AA$ ("weak" DNA-binding site). Two DNA-binding sites independently bind and release DNA in response to the signals received from the NTPase site upon translocation. The "strong" DNA-binding site that tightly binds nucleic acids via hydrogen bonding is responsible for stabilization of the DNA strand, so it could optimize processivity and also provide a stable DNA template for priming. On the other hand, the "weak" DNA-binding that transiently binds nucleic acids via loops protruding from each subunit is responsible for the directional motion forward to the junction. Consequently, reducing the interaction between NTD of protein and DNA is a potential way of loosening DNA strand, which in turn might increase helicase translocation velocity.

\section{Primase accelerates the unwinding velocity of GkDnaC helicase}

The chromosomal DNA replicases are multiprotein molecular machines. Previous studies showed that a ring-shaped hexameric DNA helicase forms a complex with either its loader or primase, resulting in the alteration of enzymatic activity [12,30,31]. To clarify how cooperativity and functional relevance between DnaC helicase and primosomal proteins in G. kaustophilus are achieved, we determined the duplex unwinding and ATP hydrolysis activities when DnaC helicase is in complex with DnaG or DnaI. Previous studies have shown that in the absence of nucleotides, $G k$ DnaC and $G k$ DnaI can form a stable complex, which facilitates ssDNA binding [12]. Here, we also observed that GkDnaC helicase forms a stable complex with $G k \mathrm{DnaG}$ primase in the presence of ATP using native PAGE and gel filtration (data not shown). As shown in Figure S6A, the unwinding activity and ATPase activity of the $G k \mathrm{DnaC}$ helicase/GkDnaG primase complex were increased 1.5- and 2-fold, respectively, as compared to those of $G k \mathrm{DnaC}$ alone. By contrast, helicase unwinding was inhibited when GkDnaC was pre-incubated with equimolar amounts of GkDnaI loader prior to the assay. The GkDnaCGkDnaI complex exhibited a lowered ATP hydrolysis rate $(18.1 \pm 5.4 \mu \mathrm{M} / \mathrm{min})$, almost half of that exhibited by $G k \mathrm{DnaC}$ alone $(32.6 \pm 10.5 \mu \mathrm{M} / \mathrm{min})$. We noted that $G k \mathrm{DnaI}$ has no detectable ATPase activity by itself under identical assay conditions (Figure S6B). It is likely that the binding of DnaI to DnaC may induce a conformational change in the ATP-binding pocket of DnaC helicase, resulting in lower ATP hydrolysis and unwinding efficiency. 
Table 2. Enzymatic activities of wild-type and mutant $G k D n a C$ helicases.

\begin{tabular}{|c|c|c|c|c|}
\hline & WT & R332A & R145A/K146A & R420A \\
\hline Mutant locations & - & CTD, $\alpha 15$ & NTD, $\alpha$-hairpin $(\alpha 7)$ & Arg finger \\
\hline$K_{D}(M)$ & $4.69 \pm 0.40 \times 10^{-8}$ & $6.64 \pm 0.90 \times 10^{-6}$ & $2.70 \pm 0.60 \times 10^{-5}$ & n.d. ${ }^{a}$ \\
\hline Unwinding efficiency (fold) & 1 & $3.67 \pm 0.85$ & $2.86 \pm 0.54$ & No activity \\
\hline ATPase activity $(\mu \mathrm{M} / \mathrm{min})$ & $32.58 \pm 10.49$ & $31.80 \pm 15.41$ & $33.95 \pm 6.58$ & No activity \\
\hline Unwinding velocity (bp/s) & $3.58 \pm 1.62$ & $7.50 \pm 2.93$ & $10.52 \pm 2.09$ & n.d. ${ }^{a}$ \\
\hline
\end{tabular}

${ }^{a}$ n.d., not detected.

doi:10.1371/journal.pone.0029016.t002

Consistent with previous studies [31,32] and as mentioned above, our bulk assay showed increases both in helicase activity and in ATPase activity of GkDnaC in the presence of $G k \mathrm{DnaG}$ primase, while reduced helicase activity was found in the presence of loader protein GkDnaI (Table 3). However, there are several factors that can lead to enhanced helicase efficiency, such as enhanced helicase processivity and enhanced helicase unwinding velocity. In addition, there is no existing model to explain why primase can stimulate helicase activity. Thus, we also measured the unwinding velocities of $G k$ DnaC- $G k \mathrm{DnaG}$ and $G k \mathrm{DnaC}-$ GkDnaI complexes using smTPM in real-time (Figure 3B). The unwinding velocity of $G k \mathrm{DnaC}-G k \mathrm{DnaG}$ was 2 -fold increased to $7.44 \pm 3.41 \mathrm{bp} / \mathrm{s}(\mathrm{N}=18)$, while no apparent unwinding process was observed for the $G k$ DnaC-GkDnaI complex within 30 minutes of recording (data not shown). Due to our design of nicked substrates, the disappearance of tethers signals the completion of the unwinding process. Therefore, the number of tethers retained at a given reaction time denotes the progress of the unwinding process. Although no unwinding velocity was determined for $G k$ DnaC-GkDnaI, we compared the unwinding activity of $G k \mathrm{DnaC}$ and its complexes by measuring the number of tethers retained in the same field-of-view at various reaction time, and presented by the percentage of disappeared bead in Figure 3E. Due to the substrate design of the single-molecule experiments, the time-course of disappeared beads directly reflects the helicase unwinding activity. For the GKDnaC-GkDnaI complex, the percentage of disappeared bead is lower than that of $G k \mathrm{DnaC}$ alone, and much lower than that of the $G k$ DnaC-GkDnaG complex. It is likely that tight binding between the GkDnaCGkDnaI complex and ssDNA $\left(\mathrm{K}_{\mathrm{D}}=47.3 \mathrm{nM}\right.$, [12]) serves as a roadblock, and makes it unfavorable for DnaC helicase to translocate on the tracking strand, the strand that helicase engages and translocates along. Our studies show that $G k \mathrm{DnaG}$ primase accelerates the rate of ATP hydrolysis of helicase resulting in faster unwinding velocity. It is possible that primase enhances the helicase unwinding velocity by increasing ATPase activity and/or by inducing a conformational change that favors rapid movement on the tracking strand. Previously report also indicated that primase can enhance the processivity of the helicase by stabilizing DNA binding and hexamer formation when it associates with the helicase [33]. The observation that the unwinding velocity of helicase could be modulated by the efficiency of ATP hydrolysis but not interaction between the tracking strand and helicase in the case of the primosomal protein, is in strong contrast to the helicase mutagenesis studies.

In summary, our ensemble-averaged and single-molecule studies on $G k$ DnaC helicase and its mutants suggest that how helicase interacts with its tracking DNA strand directly affects the unwinding process. Reducing the DNA-binding affinity of the residues located inside the central channel of the $G k \mathrm{DnaC}$ helicase efficiently enhances the unwinding efficiency and unwinding velocity without affecting the ATPase activity. Therefore, we suggested that the ring-shaped hexameric helicase might have a variety of conformations, and it can unwind more efficiently by a special conformation which is favorable for speedy translocation with a weaker ssDNA-binding ability. As expected, replication accessory proteins, such as GkDnaG primase, stimulated helicase activity. However, different from the helicase mutants, the stimulation is caused because that primase could help to regulate ATPase rate of the helicase achieving a rapider unwinding velocity. These two different modes of helicase activity modulation demonstrate that both protein-DNA interaction and proteinprotein interaction regulate the action of the replicative helicase. Apparently, however, the enhanced ATPase and helicase unwinding velocity are not sufficient to attain the replisome rate required in vivo. Other accessory proteins are thus essential to interact with $G k \mathrm{DnaC}$ helicase for further enhancement of its unwinding velocity and processivity. Future studies of the helicase activity of GkDnaC in complex with individual and sets of accessory proteins as well as that of the holoenzyme will elucidate the complexity of the regulatory network within the whole replisome machinery.

Table 3. Enzymatic activities of GkDnaC, GkDnaC-GkDnaG complex and GkDnaC-GkDnal complex.

\begin{tabular}{|c|c|c|c|}
\hline & GkDnaC & GkDnaC-GkDnaG & GkDnaC-GkDnal \\
\hline & helicase & helicase-primase & helicase-loader \\
\hline Unwinding efficiency (fold) & 1 & $1.45 \pm 0.21$ & $0.15 \pm 0.07$ \\
\hline ATPase activity $(\mu \mathrm{M} / \mathrm{min})$ & $32.58 \pm 10.49$ & $79.51 \pm 18.21$ & $18.08 \pm 5.44$ \\
\hline Unwinding velocity (bp/s) & $3.58 \pm 1.62$ & $7.44 \pm 3.41$ & ${ }_{-}^{a}$ \\
\hline
\end{tabular}

${ }^{a}$ The unwinding action is inhibited when $G k D n a C$ helicase is bound to its loader GkDnal.

doi:10.1371/journal.pone.0029016.t003 


\section{Supporting Information}

Figure S1 The rate of ATP hydrolysis of GkDnaC WT in the presence of different length of ssDNA. Reactions were initiated by addition of $G k \mathrm{DnaC}$ WT $(1 \mu \mathrm{M})$, and the rate of ATP hydrolysis was monitored by following NADH oxidation at $340 \mathrm{~nm}$. The experiments were performed using different length of ssDNA (15-mer, 30-mer and 70-mer). Except 70-mer ssDNA with random sequence, the ssDNA we used here all belong to single-stranded oligo-dT DNA (50 nM).

(TIF)

Figure S2 The initial unwinding time point determination by $1^{\text {st }}$ derivative. A-D. The raw data of helicase unwinding fork-AC90 DNA substrates, in the present of $G k$ DnaC wild-type, $G k \mathrm{DnaC}+G k \mathrm{DnaG}, G k \mathrm{DnaC}$ mutant $\mathrm{R} 332 \mathrm{~A}$ and $\mathrm{R} 145 \mathrm{~K} 146 \mathrm{~A}$, respectively. There is a recording dead time of about $20 \mathrm{~s}$ due to solution exchange and stage restabilization for imaging (shaded area). E-H. The $1^{\text {st }}$ derivative of the unwinding trace returns the initial unwinding time point. The solid red line represents the mean of the derivatives (which suppose to be zero). The dashed lines show the $95 \%$ marginal bond of the derivatives. It shows that after $270 \mathrm{sec}$, the derivatives grow over the bonds which indicate that the slope of the raw trace leaves zero at the time, that we determine as initial unwinding time point (red circle). (TIF)

Figure S3 Sequence alignment. The schematic diagram showed the sequence alignment of DnaB-like helicases from $G$. kaustophilus (GkDnaC), B. stearothermophilus (BstDnaB), Thermus aquaticus (TaqDnaB), and E. coli (EcoDnaB) that labeled with residue numbers relative to that of $G k \mathrm{DnaC}$. Residues that are completely conserved, identical and similar among family members are shaded in green, yellow and cyan, respectively. The asterisk showed the important residues (R45, R117, R120, R145, K146, K309, R330, R332, R344 and R420) that influenced DNA-bound.

(TIF)

Figure S4 Gel shift assay of increasing concentrations of GkDnaC WT. The DNA unwinding activities of protein were measured by monitoring the amount of unwound ssDNA product. The reaction was carried out in the presence of increasing

\section{References}

1. Lohman TM, Bjornson KP (1996) Mechanisms of helicase-catalyzed DNA unwinding. Annu Rev Biochem 65: 169-214.

2. Matson SW, Kaiser-Roger KA (1990) DNA Helicases. Annu Rev Biochem 59: 289-329.

3. Berger JM (2008) Nucleic acid helicases and translocases. Cell 134: 888.

4. Singleton MR, Dillingham M, Wigley DB (2007) Structure and Mechanism of Helicases and Nucleic Acid Translocases. Annu Rev Biochem 76: 23-50.

5. Patel SS, Picha KM (2000) Structure and function of hexameric helicases. Annu Rev Biochem 69: 651-697.

6. Lo Y-H, Tsai K-L, Sun Y-J, Chen W-T, Huang C-Y, et al. (2009) The crystal structure of a replicative hexameric helicase DnaC and its complex with singlestranded DNA. Nucleic Acids Res 37: 804-814.

7. Yodh JG, Schlierf M, Ha T (2010) Insight intohelicasemechanismand function revealed through single-molecule approaches. O Rev Biophys 43: 185-217.

8. Johnson DS, Bai L, Smith BY, Patel SS, Wang MD (2007) Single-Molecule Studies Reveal Dynamics of DNA Unwinding by the Ring-Shaped T7 Helicase. Cell 129: 1299-1309.

9. Lionnet T, Spiering MM, Benkovic SJ, Bensimon D, Croquette V (2007) Realtime observation of bacteriophage T4 gp41 helicase reveals an unwinding mechanism. Proc Natl Acad Sci U S A 104: 19790-19795.

10. Ribeck N, Kaplan DL, Bruck I, Saleh OA (2010) DnaB Helicase Activity Is Modulated by DNA Geometry and Force. Biophys J 99: 2170-2179.

11. Manosas M, Spiering MM, Zhuang Z, Benkovic SJ, Croquette V (2009) Coupling DNA unwinding activity with primer synthesis in the bacteriophage T4 primosome. Nat Chem Biol 5: 904-912. amounts of purified proteins as indicated $(0.1,0.15,0.25,0.5,1,2$ and $4 \mu \mathrm{M})$. To normalize these values, the percentage of product was calculated using the equation, \%Unwound $=\left(\% \mathrm{U}_{\mathrm{S}}-\% \mathrm{U}_{0}\right) /$ $\left(\% \mathrm{U}_{100} \mathrm{G}-\% \mathrm{U}_{0}\right)$ (detail in materials and methods). Two independent experiments are shown here and are represented as red and blue lines (right panel).

(TIF)

Figure S5 The rate of ATP hydrolysis of GkDnaC under different molar ratio of protein to ssDNA. The rate of ATP hydrolysis is proportional to the rate of the decrease in absorbance at $\mathrm{OD}_{340}$, and it can be calculated according to the formula: $\Delta \mathrm{A}_{340} /$ time $\left(\mathrm{s}^{-1}\right) \times 9820=$ rate of ATPase $(\mu \mathrm{M} / \mathrm{min})$. Reactions were initiated by the addition of $1 \mu \mathrm{M}$ GkDnaC WT (per monomer) into the mixture in the presence of 15-mer singlestranded oligo-dT DNA (50 nM or $10 \mu \mathrm{M}$ ). Two independent experiments are shown here in different molar ratio system.

(TIF)

Figure S6 Unwinding and ATPase activity of GkDnaC were affected when GkDnaG was associated with primosomal protein. A. The effect of primosomal protein on helicase unwinding efficiency. The abscissa shows varying enzyme and complex formation, which is correlated to the unwinding efficiency (fold) shown on the top of each bar. B. The effect of primosomal protein on ATP hydrolysis of $G k \mathrm{DnaC}$. The rate of ATP hydrolysis was calculated from the rate of change in absorbance at $340 \mathrm{~nm}$ due to oxidation of NADH. Data represent the average of three independent experiments.

(TIF)

\section{Acknowledgments}

We are grateful to Jui-Yun Chang for performing pilot single-molecule experiments.

\section{Author Contributions}

Conceived and designed the experiments: Y-JS H-WL C-DH. Performed the experiments: Y-HL S-WL. Analyzed the data: Y-JS H-WL C-DH. Contributed reagents/materials/analysis tools: Y-JS H-WL. Wrote the paper: H-WL C-DH.

12. Tsai K-L, Lo Y-H, Sun Y-J, Hsiao C-D (2009) Molecular Interplay between the Replicative Helicase DnaC and Its Loader Protein DnaI from Geobacillus kaustophilus. J Mol Biol 393: 1056-1069.

13. Spies M, Dillingham MS, Kowalczykowski SC (2005) Translocation by the RecB Motor Is an Absolute Requirement for $\chi$-Recognition and RecA Protein Loading by RecBCD Enzyme. J Biol Chem 280: 37078-37087.

14. Datta K, Neumann RD, Winters TA (2003) A protocol for separation and isolation of small and/or large DNA fragments with high yield using CL4B Sepharose. Anal biochem 317: 284-287.

15. Chu J-F, Chang T-C, Li H-W (2010) Single-Molecule TPM Studies on the Conversion of Human Telomeric DNA. Biophys J 98: 1608-1616.

16. Fan H-F, Li H-W (2009) Studying RecBCD helicase translocation along chiDNA using tethered particle motion with a stretching force. Biophys J 96: 1875-1883.

17. Pouget N, Dennis C, Turlan C, Grigoriev M, Chandler M, et al. (2004) Singleparticle tracking for DNA tether length monitoring. Nucleic Acids Research 32: e73.

18. Nelson PC, Zurla C, Brogioli D, Beausang JF, Finzi L, et al. (2006) Tethered Particle Motion as a Diagnostic of DNA Tether Length. J Phys Chem B 110: $17260-17267$.

19. Farge Gr, Holmlund T, Khvorostova J, Rofougaran R, Hofer A, et al. (2008) The N-terminal domain of TWINKLE contributes to single-stranded DNA binding and DNA helicase activities. Nucleic Acids Res 36: 393-403.

20. Marrione PE, Cox MM (1995) RuvB Protein-Mediated ATP Hydrolysis: Functional Asymmetry in the RuvB Hexamer. Biochemistry 34: 9809-9818. 
21. Bird LE, Pan H, Soultanas P, Wigley DB (2000) Mapping Protein-Protein Interactions within a Stable Complex of DNA Primase and DnaB Helicase from Bacillus stearothermophilus. Biochemistry 39: 171-182.

22. Manosas M, Xi XG, Bensimon D, Croquette V (2010) Active and passive mechanisms of helicases. Nucleic Acids Res 38: 5518-5526.

23. Oijen AMv, Loparo JJ (2010) Single-Molecule Studies of the Replisome. Annu Rev Biophys 39: 429-448.

24. Singleton MR, Sawaya MR, Ellenberger T, Wigley DB (2000) Crystal Structure of T7 Gene 4 Ring Helicase Indicates a Mechanism for Sequential Hydrolysis of Nucleotides. Cell 101: 589-600.

25. Jezewska MJ, Kim US, Bujalowski W (1996) Binding of Escherichia coli primary replicative helicase DnaB protein to singlestranded DNA. Long-range allosteric conformational changes within the protein hexamer. Biochemistry 35: 2129-2145.

26. Jezewska MJ, Rajendran S, Bujalowski W (1998) Functional and structural heterogeneity of the DNA binding site of the Escherichia coli primary replicative helicase DnaB protein. J Biol Chem 273: 9058-9069.
27. Bujalowski W, Jezewska MJ (1995) Interactions of Escherichia coli primary replicative helicase DnaB protein with single-stranded DNA. The nucleic acid does not wrap around the protein hexamer. Biochemistry 34: 8513-8519.

28. Thomsen ND, Berger JM (2009) Running in Reverse: The Structural Basis for Translocation Polarity in Hexameric Helicases. Cell 139: 523-534.

29. Enemark EJ, Joshua-Tor L (2006) Mechanism of DNA translocation in a replicative hexameric helicase. Nature 442: 270-275.

30. Bailey S, Eliason WK, Steitz TA (2007) Structure of Hexameric DnaB Helicase and Its Complex with a Domain of DnaG Primase. Science 318: 459-463.

31. Soultanas P (2002) A functional interaction between the putative primosomal protein DnaI and main replicative DNA helicase DnaB in Bacillus. Nucleic Acids Res 30: 966-974.

32. Wahle E, Lasken RS, Kornberg A (1989) The dnaB-dnaC replication protein complex of Escherichia coli. II. Role of the complex in mobilizing dnaB functions. J Biol Chem 264: 2469-2475.

33. Frick DN, Richardson CG (2001) DNA Primases. Annu Rev Biochem 70: 39-80. 